\title{
In Vitro Clonal Propagation of Musa Sp. Cultivar Gopi: A Palatable Banana of Tripura, India
}

\author{
Rabindra Kumar Sinha ${ }^{1, *}$, Puja Rani Saha ${ }^{1}$, Anath Bandhu Das ${ }^{2}$, Satya Narayan Jena ${ }^{3}$, \\ Sangram Sinha ${ }^{1}$ \\ ${ }^{1}$ Cytogenetics and Plant Biotechnology Lab., Department of Botany, Tripura University, Suryamaninagar, India \\ ${ }^{2}$ Department of Botany, Utkal University, Bhubaneswar, India \\ ${ }^{3}$ Genetics and Molecular Biology, Council of Scientific and Industrial Research-National Botanical Research Institute, Lucknow, India
}

Email address:

khsinhark@yahoo.co.in (R. K. Sinha)

${ }^{*}$ Corresponding author

\section{To cite this article:}

Rabindra Kumar Sinha, Puja Rani Saha, Anath Bandhu Das, Satya Narayan Jena, Sangram Sinha. In Vitro Clonal Propagation of Musa sp. Cultivar Gopi: A Palatable Banana of Tripura, India. American Journal of Plant Biology. Vol. 3, No. 1, 2018, pp. 12-16.

doi: 10.11648/j.ajpb.20180301.13

Received: April 18, 2018; Accepted: May 3, 2018; Published: May 30, 2018

\begin{abstract}
The present investigation has been concentrated on the establishment of an efficacious method for in vitro regeneration of a Musa cultivar Gopi of Tripura. Focus has been greatly anchored to the sterilization procedure for assuring successful aseptic culture establishment of this cultivar. The culture protocol for shoot induction and proliferation comprised of four different treatments in basal MS medium fortified with 6-Benzylaminopurine of varying concentrations. The optimum response was encountered in the treatment of BAP in MS medium at concentration of $8 \mathrm{mg}^{-1}$ for shoot bud induction and multiplication. Subsequent shoot growth and multiplication was achieved through repeated subcultures in media containing lower concentration $\left(4 \mathrm{mg} \mathrm{l}^{-1}\right)$. Root initiation was stimulated and brought about by treatments with two different concentrations of Indole butyric acid. The best rooting was manifested in treatment with IBA at concentration of $2 \mathrm{mg}^{-1}$. The present study is an approach towards successful establishment of a simple and rapid clonal propagation of a potent Musa cultivar Gopi having traditional and commercial value from this region of Tripura, North-east India.
\end{abstract}

Keywords: In Vitro Regeneration, Musa Cultivar Gopi, Benzylaminopurine, Indole Butyric Acid, Clonal Propagation, Tripura

\section{Introduction}

Banana is one of the chief fruit crops for global food security and economy ranking fourth in the most crucial food commodities worldwide in terms of gross production [1-3]. The fruit is a potent source of essential nutrients rich in carbohydrates, proteins, vitamins and minerals. Biotechnological refinement in banana is now obvious and widely practiced for clonal selection and propagation leading to mass production of elite clones. Thus, in vitro techniques prove to be beneficial in order to supplement the conventional breeding processes and to overcome the constraints inflicted by pests and pathogens [4]. Tripura inhabits a rich resource of wild and cultivated Musa varieties. One of the cultivars with immense socio-economic potential in this part of north-eastern region is Gopi Kola. This specific cultivar holds a position of huge importance not only as an edible fruit crop but also utilized for sacred purposes by local communities of Tripura since many years. Gopi Kola thus occupies a rank of significant relevance as a promising fruit in the context of edibility, ethnicity and economic grounds in this region. But, the plantation and cultivation practices of this cultivar do not satisfactorily meet the demands of consumption in domestic markets. So, the supply of this cultivar requires enhancement in its mode of productivity to optimally encounter the desire of the consumers. This can be successfully achieved by in vitro practices for mass multiplication of this socio-economic cultivar. Several pests and pathogens are known to endanger the production of banana cultivars. The improvement of banana micropropagation techniques has been a crucial priority in Musa research throughout the last two decades. Several 
techniques and protocols have now been well attained and materialised in the dimension of banana micropropagation. This has been achieved using different explants like shoot tip [5], male floral apices [6]. Somatic embryogenesis and regeneration in liquid medium is also reported for banana [79]. Successful in vitro micropropagation technique was also reported for Musa acuminata cv. Vaibalhla through immature male flowers [10]. Though numerous approaches towards in vitro propagation of many Musa cultivars have been made, the present attempt emphasizes on the cultivar Gopi Kola for generating a simple, rapid and effective protocol highlighting on the sterilization steps for the in vitro propagation of such a leading traditional and economic cultivar of Tripura, North east India. The present investigation shall also be an addition to the in vitro techniques for banana micropropagation.

\section{Materials and Methods}

Banana cultivar Gopi, a leading traditional as well as commercial cultivar of Tripura was the investigating subject. The source materials used for aseptic culture of this cultivar were healthy rhizome explants. Young suckers were collected from Melaghar, West district of Tripura and explants were obtained by removing the pseudostem portion above the rhizome collar using a sanitized knife and peeling off the outer culms of rhizome. The aseptic preparation of explants is one of the most crucial steps concerning the banana culture initiation and progress. The explants were initially sterilized through a series of sterilization steps using cleansing and disinfecting agents accompanied by several washing phases. The trimmed rhizomes were thoroughly washed under running tap water to remove the surface detritus followed by cleansing with 5\% Savlon solution for $1 \mathrm{hr}$. Explants were then rinsed off with water followed by treatment with disinfectant solution of bavistin of $0.5 \%$ for $4 \mathrm{hrs}$. The same were once again rinsed off under splashing water and subsequently transferred to a solution of activated charcoal and kept overnight. This was followed by washing with water and finally trimmed with a sterilized scalpel to about $5 \times 5$ $\mathrm{cm}^{2}$ size. Final Surface sterilization of the explants was completed after treatment with $0.1 \% \mathrm{HgCl}_{2}$ solution for 5 minutes followed by serial washing in three changes of sterile distilled water in the laminar hood. This was followed by the final aseptic trimming of the rhizome explants into three to four suitable pieces without much wounding for inoculation. Each piece of the explant was used as inoculum for aseptic culture establishment. Culture media used in the present study was MS basal medium with $30 \mathrm{gl}^{-1}$ sucrose and $9 \mathrm{~g} \mathrm{l}^{-1}$ agar and adjusted to $\mathrm{p}^{\mathrm{H}} 5.8$ before autoclaving at $120^{\circ} \mathrm{C}$ for $20 \mathrm{~min}$. In vitro morphogenic responses and adventitious shoot bud proliferation were investigated with different experimental sets of media supplemented with different concentrations of cytokinin BAP $\left(2 \mathrm{mg} \mathrm{l}^{-1}, 4 \mathrm{mg} \mathrm{l}^{-1}, 6 \mathrm{mg} \mathrm{l}^{-1}\right.$ and $\left.8 \mathrm{mg}^{-1}\right)$. All cultures were incubated at $26 \pm 2^{\circ} \mathrm{C}$. Successful establishment of culture through multiple shoot formation was achieved by regular subculturing at 28 and 42 days interval on freshly prepared same medium. Black tissues were removed prior to subculturing. Number of shoots and shoot size were noted at every subculture. A mean of five replicates were taken under consideration for record of shoot multiplication and elongation. Successive subcultures were performed for obtaining multiple shoots. After developing healthy elongated shoots in culture, they were transferred to MS medium supplemented with different concentrations of IBA viz. $2 \mathrm{mg} \mathrm{l}^{-1}, 4 \mathrm{mg} \mathrm{l}^{-1}$. For the purpose of hardening, the rooted plantlets were encountered with subtle exposure to sunlight for a week; then plantlets were carefully washed with tap water to remove the adherent traces of agar and subsequently transferred to water medium for another week. The same were later on transferred to pots containing a mixture of sterilized soil, soilrite and sand in 2:1:1 ratio. They were kept under shade-house at relative humidity of $80-90 \%$. The plants were thus acclimatized and finally transferred to natural field. Statistical analysis by Two-way ANOVA was performed followed by Fishers' Least significant difference test to reveal any possible significant difference existing between treatments with BAP and duration of culture as well. Mitotic chromosomes were studied following the standard protocol of Rekha and Hiramath [11] with few modifications and subjected to subsequent standard method of squashing technique [12].

\section{Results and Discussion}

The present study was attempted to develop an effective protocol for regeneration of a prime traditional and commercial banana variety of Tripura popularly known as Gopi Kola by the local people. The study was conducted with keen approach towards effective sterilization and establishing successful aseptic culture. The use of different sterilants like Savlon and $0.5 \%$ Bavistin solution has been optimized as surface disinfectants for certain durations in the effective preparation of explants. Use of activated charcoal for prolonged duration has greatly reduced the exudation problem of rhizome explants. In addition, final treatment of explants with $0.1 \%$ mercuric chloride solution for five minutes was found to be effective sterilant for aseptic culture establishment of the cultivar as reported in many other in vitro cultures of banana $[13,14]$.

The effect of different concentrations of BAP $\left(2 \mathrm{mg} \mathrm{l}^{-1}\right.$, $4 \mathrm{mg} \mathrm{l}^{-1}, 6 \mathrm{mg} \mathrm{l}^{-1}$ and $8 \mathrm{mg} \mathrm{l}^{-1}$ ) in MS medium was examined for the productive in vitro shoot induction and propagation from rhizome explants (Figure 1 A). Cultured explants produced visible morphogenic response initially as a swelling appearance with greenish colour after 10 to 15 days of inoculation along with occasional blackening at the base due to exudation. Shoot induction was relatively good at higher concentrations of BAP in comparison to treatment with low concentration. Incubation of morphogenic explants for prolonged duration in the same medium however continued to induce shoot bud formation. The best response concerning shoot induction at initial cultures was recorded in medium containing BAP with $8 \mathrm{mg}^{-1}$ where as medium with BAP at $2 \mathrm{mg}^{-1}$ yielded lowest response on shoot induction (Table 1). 
Shoot multiplication was notably productive after first subculture of 28 days in medium containing high concentrations of BAP at $6 \mathrm{mg} \mathrm{l}^{-1}$ and $8 \mathrm{mg} \mathrm{l}^{-1}$ (Figure B). Subsequent subculture of 42 days old morphogenic explants in MS medium fortified with lower concentrations of BAP at $2 \mathrm{mg} \mathrm{l}^{-1}$ and $4 \mathrm{mg} \mathrm{l}^{-1}$ supported shoot elongation as well as generation of limited number of new shoot buds (Figure C). In general, incidence of shoot proliferation and multiplication gradually expanded with the increase of subcultures in the same medium for almost all the treatments with BAP. Emergence of new shoot buds and shoots with varying sizes (Figure D) were continuously achieved by repeated subculturing of the proliferating shoot cluster in pieces in alternate high and low concentrations of BAP supplemented MS media. Maximum number of shoots was recorded in the range of 6-9 during 42 days old culture (Table 1). Optimum shoot elongation was also recorded in subsequent cultures treated with BAP at $4 \mathrm{mg} \mathrm{l}^{-1}$ and $6 \mathrm{mg} \mathrm{l}^{-1}$ (Table 1). Elongated healthy shoots subcultured in medium containing $2 \mathrm{mg} \mathrm{l}^{-1} \mathrm{IBA}$ proved to be the most effective treatment for rooting (Figure E). The regenerated plantlets were subsequently transferred to liquid medium without any plant growth regulator and vitamins and kept for 6-7 days followed by hardening through transfer to clean tap water medium (Figure F). This was followed by successful transfer of the regenerated clones in potted soil mix (Figure G) and finally transferred to open natural condition (Figure H). Morphologically, no detectable variation was observed in the regenerated clones. Hardening of the in vitro clones revealed $100 \%$ survival upon transfer to soil.

Table 1. Effect of cytokinin BAP on shoot bud induction and multiplication of Musa cultivar Gopi.

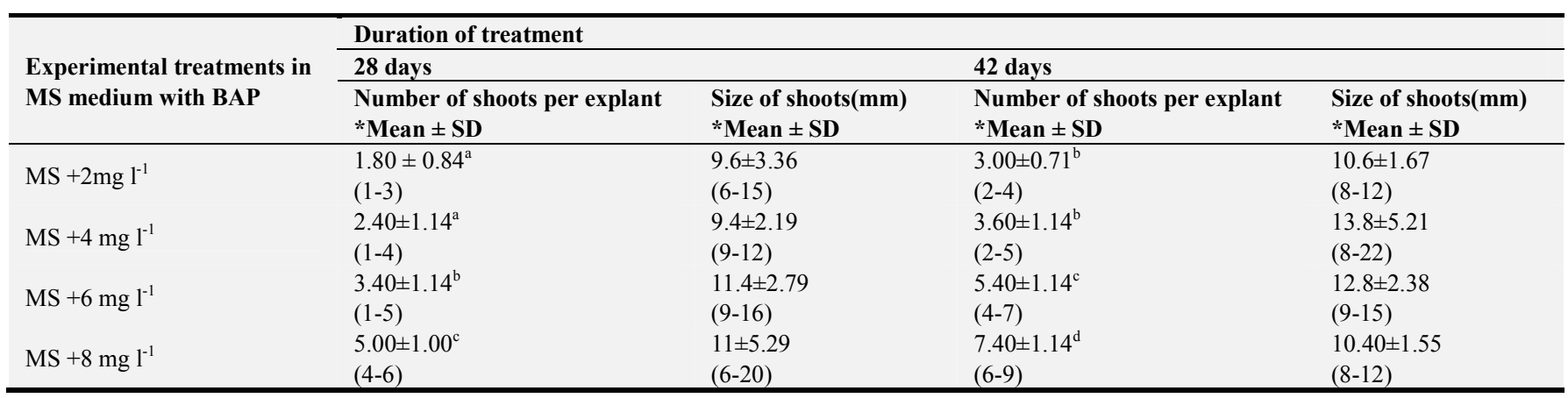

Least Significant Difference $(0.05 \%)=0.630$; different letters above indicate significant differences between each other; within parenthesis ( ) range of number of shoots and shoot sizes; *mean of five replicates; $\mathrm{SD}=$ Standard Deviation.

Table 2. Two-way ANOVA for shoot multiplication in different experimental treatments.

\begin{tabular}{lllllll}
\hline Source of variation & SS & df & MS & F-value & P-value \\
\hline Experimental treatments & 17.12 & 3 & 5.706667 & 31.7037 & 0.008994 \\
Duration & 5.78 & 1 & 5.78 & 32.11111 & 0.010885 \\
Error & 0.54 & 3 & 0.18 & & 10.1279628 \\
Total & 23.44 & 7 & & \\
\hline
\end{tabular}

$\mathrm{SS}=$ Source of variation, $\mathrm{df}=$ degrees of freedom, $\mathrm{MS}=$ Mean square error.

Two-way ANOVA revealed significant differences between experimental treatments with BAP as well as duration of culture for number of shoot differentiation. Results indicate treatment with higher concentrations of BAP induce significant impact on shoot initiation and multiplication (Table 1 and Table 2). Moreover, duration of culture also play crucial role for shoot bud promotion in all treatments (Table 2). The optimum and effective treatment of cytokinin for fruitful shoot multiplication of the cultivar Gopi was recorded in MS medium supplemented with BAP $8 \mathrm{mg} \mathrm{l}^{-}$ ${ }^{1}$ during 42 days old culture (Table 1). However, previous reports on banana micropropagation demonstrated the use of MS medium fortified with BAP and IAA proving as the effectual combination and providing with the desired outcomes concerning rapid shoot initiation [15-17]. Several reports on tissue culture of banana have also been reported by different workers using cytokinin as a principal growth regulator for induction and multiplication of shoot bud formation [5, 18-22]. Superiority of BAP over kinetin along with IBA for in vitro multiplication of banana has been recorded [19]. The effectiveness of BAP over other cytokinins in inducing multiplication of shoot tip cultures was also reported [23-26]. The present study clearly indicated suitability of cytokinin BAP at different concentrations for efficient induction and multiplication of healthy shoot buds and shoots. Subsequent rooting of the in vitro regenerants was achieved in media containing IBA as principal hormone.

Existence of genetic uniformity was also confirmed by cytological study of somatic chromosome number of the regenerated plant. The regenerated plant revealed $2 n=3 x=33$ somatic chromosomes (Figure 1) thus exhibiting similar chromosome count as that of the parent plant. 


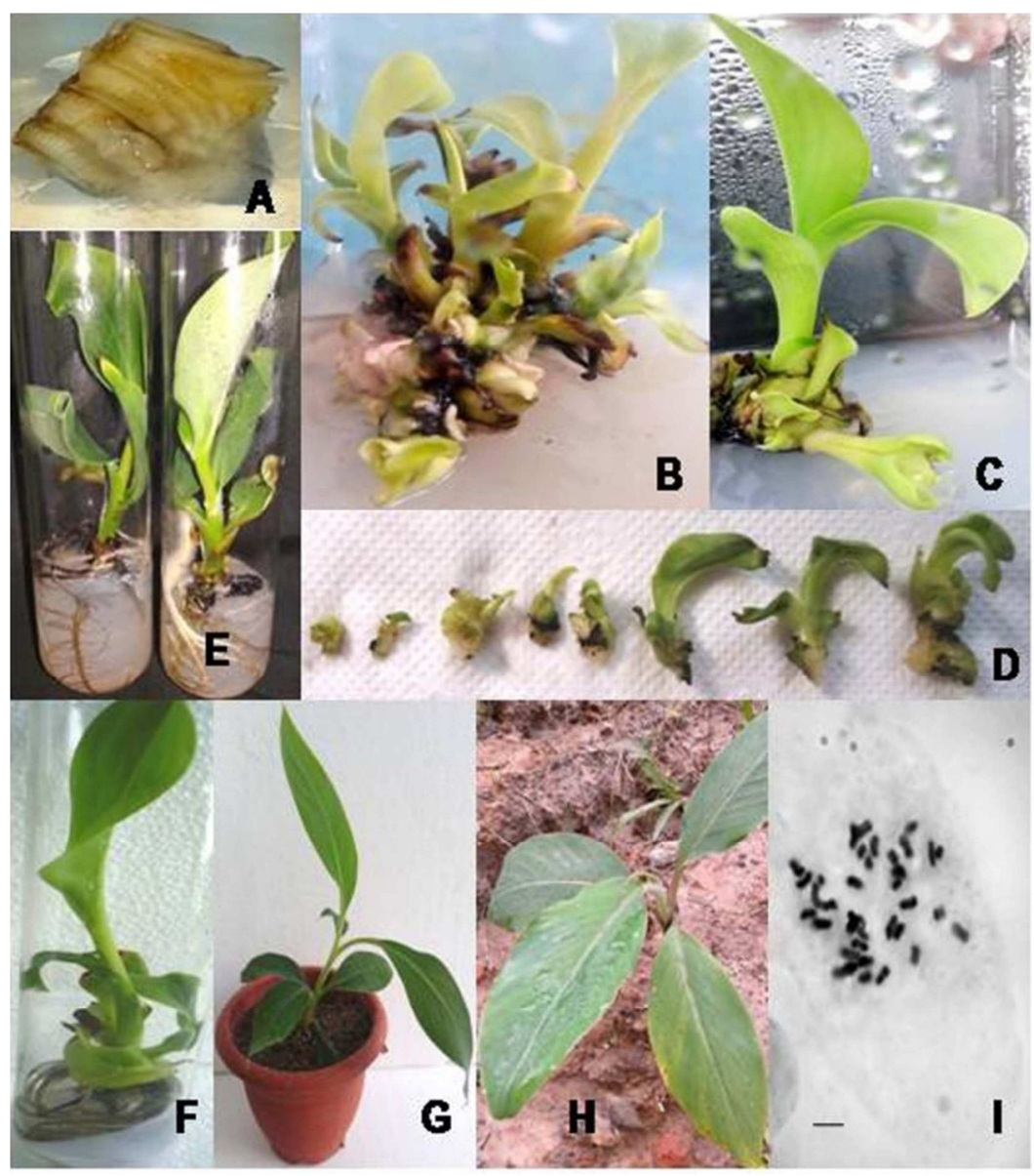

Figure 1. In vitro clonal propagation of Musa cultivar Gopi. A) Sucker rhizome explant used in culture; B) Shoot bud initiation and multiplication in MS medium with $\left.8 \mathrm{mg} l^{-1} B A P ; C\right)$ Elongated Shoots; D) Range of shoot sizes in a proliferating shoot cluster; E) Root induction and growth in rooting medium with $\left.2 m g l^{-1} I B A ; F\right)$ Hardening of healthy banana plantlet in water medium; G) Plantlet transferred to potted soil mix containing soil, soilrite and sand; $H$ ) Successful transfer of plantlet to field condition; I) Somatic chromosome number of regenerated banana cultivar, $2 n=3 x=33($ Scale bar $=2 \mu)$.

The present study reports a rapid in vitro method for clonal propagation of Musa cultivar Gopi within the time frame of 14 weeks of culture. Therefore, this method would serve to greatly enhance regeneration efficiency of this cultivar and meet the need for its extensive production thus establishing the demand and supply equilibrium of Gopi cultivar in Tripura.

\section{Conclusion}

The present study effectively generated a simple and rapid in vitro propagation method of a significant banana cultivar Gopi of Tripura. Establishment of aseptic culture was successful through the optimization in the use of different sterilants for explant preparation. Shoot bud induction and multiplication was effective in MS media supplemented with high concentration of BAP. Proliferation of new shoot buds was achieved through repeated subcultures in alternate high and low BAP containing media. The present in vitro protocol can serve as a useful technique for the rapid and large scale production of the Gopi cultivar that can be utilised in food security and economic benefits for the people of this northeastern region. Moreover, the same protocol may also be implemented in genetic enhancement programmes of this very less explored important Musa cultivar of Tripura.

\section{Acknowledgements}

The authors are grateful to the Department of Biotechnology, Govt. of India for providing financial support in the form of a project (No. BCIL/NER-BPMC/2018/245) for carrying out this research work.

\section{References}

[1] INIBAP. International network for the improvement of banana and plantain. Annual Report, Montpellier, France. 1998.

[2] FAO. On-line database of the Food and Agricultural Organization of the United Nations.

http://apps.fao.org/default.html.2001.

[3] B. Aquil, T. J. Arif, N. B. Sarin, Q. M. R. Haq "Micro propagation and genetic transformation of banana for crop improvement and sustainable agriculture" J of Crop Sci, vol. 3(2) pp. 64-77. 2012.

[4] L. Tripathi "Genetic engineering for improvement of Musa protection in Africal" Afr J Biotechnol, vol. 2(12), pp. 503508. 2003. 
[5] S. S. Cronauer, A. D. KriKorian "Multiplication of Musa from excised stem tips" Ann Bot (London), vol. 53, pp. 321-328. 1984.

[6] R. DoreSwamy, L. Sahijram"Micropropagation of banana from male floral apices culture in vitro"Sci Hortic, vol. 40(3) pp. 181-188. 1989.

[7] F. J. Novak, R. Afza, M. Van Duren, M. Perea-Dallos, B. V. Conger, T. Xiolang "Somatic embryogenesis and plant regeneration in suspension cultures of desert (AA, AAA) and cooking(AAB) bananas (Musa spp.)" Biotechnology, vol. 7, pp. $154-159.1989$.

[8] F. X. Cote, R. Domergue, S. Monmarson, J. Schwendiman, C. Teisson, J. V. Escalant"Embryogenic cell suspension from the male flower of Musa AAA cv. Grand nain"Physiol Plant, vol. 97, pp. 285-290. 1996.

[9] M. A. K, Azad, M. N. Amin "Rapid clonal propagation of banana (Musa spp.) using in vitro culture of floral bud apex" Plant tissue cult, vol. 1, pp. 1-9. 2001.

[10] L. Hrahsel, A. Basu, L. Sahoo "In vitro Propagation and assessment of the genetic fidelity of Musa acuminate (AAA) cv. Vaibalhla Derived from Immature male Flowers" Appl Biochem Biotechnol, vol. 172, pp. 1530-1539. 2014.

[11] A. Rekha, S. C. Hiremath "Chromosome studies and Karyotype analysis of some triploid banana(Musa species) cultivars of AAA genomic group" J Hortl Sci, vol. 3(1), pp. 30-34. 2008.

[12] A. K. Sharma, A. Sharma. "Chromosome techniques: theory and practice". Butterworths. London. 1965.

[13] N. Banerjee, A. K. Sharma "In vitro response as a reflection of genomic diversity in long-term cultures of Musa" Theoritical and Applied Genetics, vol. 76, pp. 733-736. 1988.

[14] U. Habiba, S. Reja, M. L. Saha, M. R. Khan, S. Hadiuzzaman"Endogenous bacterial contamination during in vitro culture of table banana: Identification and prevention" Plant Tissue Cult, vol. 12, pp. 117-124. 2002.

[15] S. Ahmed, A. Sharma, A. K. Singh, V. K. Wali, P. Kumari "In vitro multiplication of banana (Musasp.)cv. Grand Naine" Afr J Biotechnol, vol. 13(37), pp. 2696-2703. 2014.

[16] P. Karule, V. Dalvi, A. Kadu, R. Chaudhari, V. R. SubramaniamA. B. Patil "A commercial micropropagation protocol for virupakshi (AAB) banana via apical meristem" Afr J Biotechnol, vol. 15(11), pp. 401-407. 2016.

[17] K. Maharani, S. Beura, P. S. Munsi "A Fast Protocol for in vitro cloning of Banana (Musaacuminata) cv. Amritpani”'Int J Curr Microbiol, App Sci, vol. 6(10), pp. 586-594. 2017.

[18] R. L. Jarret "In vitro propagation and genetic conservation of bananas and plaintains" In IBPGR Advising committee in vitro storage, report of the third meeting (appendix), IBPGR, Rome, Italy. 1986.

[19] D. Vuylsteke, Shoot tip culture for the propagation, conservation and exchange of Musa germ plasm. IBPGR. Rome. 1989.

[20] W. C. Wong "In vitro propagation of banana (Musa spp.): Initiation, proliferation and development of shoot tip cultivars on define media" Plant cell Tissue and Organ Cult, vol. 6, pp. 159-166. 1986.

[21] G. Arinative, P. R., Rubaihago, M. J. S. Magambo "Proliferation rate effects of cytokinins on banana(Musaspp) Cultivars"Sci Hortic, vol. 86, pp. 13-21. 2000.

[22] M. Sheidai, H. Aminpoor, Z. Noormohammadi, F. Farahani "Genetic variation induced by tissue culture in Banana (Musa acuminate L.) cultivar Cavandish Dwarf Masoud" Gene Conserve, vol. 9, pp. 1-10. 2009.

[23] F. Farahani, H. Aminpoor, M. Sheidai, Z. Noormohammadi, M. H. Mazinan "An improved systemfor in vitro propagation of banana (Musa acuminate L.) cultivars" Asian J of Plant Sci, vol. 7, pp. 116-118. 2008.

[24] J. N. Buah, E. Danso, K. J. Taah, E. A. Abole, E. A. Bediako, J. Asieidu, R. Baidoo "The effects of different concentrations of cytokinins on the in vitro multiplication of plaintain (Musa sp.)" Biotech, vol. 9(3), pp. 343-347. 2010.

[25] N. Jafari, R. Y. Othman, N. Khalid "Effect of benzyl amino purine (BAP) pulsing on in vitro multiplication of Musa acuminata (banana)" Asian J of Plant Sci Res, vol. 10, pp. 2446-2454. 2010.

[26] J. Lohidas, D. Sujin "Effect of growth hormones in the micropropagation of Banana cv. Matti" Plant Archives, vol. 15 (1), pp. 307-314. 2015. 